\title{
Emotionserleben, Emotionsregulation und strukturelle Störung bei Jugendlichen
}

\author{
Eva Huber · Astrid Bock · Miriam Hans · Elke Wieser · Maria Steinmayr-Gensluckner · Kathrin Sevecke • \\ Cord Benecke
}

Eingegangen: 4. November 2015 / Angenommen: 21. Mai 2016 / Online publiziert: 22. Juni 2016

(c) Der/die Autor(en) 2016. Dieser Artikel ist eine Open-Access-Publikation.

\begin{abstract}
Zusammenfassung Das Integrationsniveau psychischer Struktur steht mit psychischer Krankheit, sowie den Bereichen Emotionserleben und Emotionsregulation in engem Zusammenhang. Es lassen sich hierin basale Dimensionen vermuten, deren differenzierte Betrachtung wichtig ist, um zwischen einem passageren Geschehen und strukturellen Defiziten entscheiden zu können. Eine umfassende strukturelle Diagnostik wäre im Kinder- und Jugendpsychiatrischen Bereich wünschenswert, ist jedoch mit einem erheblichen Zeitaufwand verbunden, weshalb eine Umsetzung im klinischen Alltag oftmals nicht möglich ist. Ziel dieser Studie ist es, ein im klinischen Alltag ökonomisch durchführbares „Risiko-Screening“ zu erproben. So wurde anhand von 51 OPD-KJ Interviews mit Jugendlichen zwischen 14 und 17 Jahren eine Einschätzung des Strukturniveaus getätigt. Diese Jugendlichen füllten zusätzlich zwei Fragebögen aus: einen Fragebogen zum Emotionserleben und zur Emotionsregulation (EER) und den Fragebogen zur Persönlichkeitsorganisation (IPO-16). In
\end{abstract}

Diese Arbeit ist Teil des Leitthemas „Psychotherapie mit Kindern und Jugendlichen“.

\section{E. Huber $(\bowtie)$}

Institut für Psychologie, Universität Innsbruck,

Innrain 52, 6020 Innsbruck, Österreich

E-Mail: Eva.Huber@uibk.ac.at

\section{A. Bock $\cdot$ K. Sevecke}

Universitätsklinik für Kinder- und Jugendpsychiatrie,

Medizinische Universität Innsbruck, Innsbruck, Österreich

\section{Hans · C. Benecke}

Institut für Psychologie, Universität Kassel, Kassel, Deutschland

\section{E. Wieser · M. Steinmayr-Gensluckner}

Universitätsklinik für Kinder- und Jugendpsychiatrie, Tirol Kliniken Innsbruck, Innsbruck, Österreich der vorliegenden Arbeit wurde getestet, ob durch die Ergebnisse der beiden Selbstbeurteilungsverfahren vorhergesagt werden kann, bei welchen Jugendlichen weiterführende Strukturdiagnostik indiziert ist. Die Ergebnisse zeigen, dass das reduzierte Erleben positiver Emotionen und die Neigung, auf subjektiv als schwierig erlebte Gefühle mit einem Blackout zu reagieren, knapp $60 \%$ der strukturell beeinträchtigten Jugendlichen korrekt zuordnete. Vierzig Prozent der strukturell vulnerablen Jugendlichen blieben jedoch durch dieses Screening unerkannt. Aus den Ergebnissen ist zu schließen, dass ein auf Selbstbeschreibungsverfahren beruhendes Screening im Rahmen einer ausführlichen Diagnostik zu Beginn der Behandlung im jugendpsychiatrischen Bereich nicht ohne zusätzliches klinisches Expertenurteil empfohlen werden kann.

Schlüsselwörter Jugendliche · Emotionserleben . Emotionsregulation · Strukturniveau $\cdot$ EER $\cdot$ OPD-KJ

Experience of Emotions, Emotion Regulation, and Impaired Structural Integration in Adolescents

Summary The level of structural integration, as well as the experience and regulation of emotions, are closely linked to mental illness. The differentiated consideration of these basic dimensions may therefore allow for a distinction between temporary clinical phenomena and more serious structural impairment. Especially in the department of childhood and adolescent psychiatry, it would thus be desirable to dispose of extensive diagnostics of structural impairment. However, this diagnostics takes up a considerable amount of time, which prevents the implementation in daily clinical practice. The aim of this study is to try out an economically practicable "risk-screening" for daily clinical practice. Therefore, 51 OPD-CA inter- 
views were conducted with adolescents aged 14 to 17 years. In addition, the adolescents filled out two questionnaires: the questionnaire for the assessment of emotional experience and emotion regulation (EER) and the Inventory of Personality Organization (IPO). Logistic regression was used to estimate whether the results of self-report questionnaires predicted structural impairment. The results show that reduced experience of positive emotions, and the tendency to react with a black-out to the experience of subjectively difficult emotions, predicted just under $60 \%$ of structurally impaired adolescents. Forty percent of the adolescents with structural vulnerability however remained unnoticed by the screening. Overall, the results indicate that a screening, which is merely based on self-report questionnaires, is not sufficient for extensive diagnostics of structural impairment in childhood an adolescent psychiatry, and cannot be a substitute for the judgement of clinical experts.

Keywords Adolescents · Emotion experience - Emotion regulation - Level of structural integration - EER . OPD-CA

\section{Einleitung}

Emotionales Erleben und die Fähigkeit, Emotionen zu regulieren, spielen für die Entstehung und Aufrechterhaltung psychischer Störungen eine entscheidende Rolle (Barlow et al. 2011; Barnow 2012; Benecke 2014; Berking et al. 2008). Schwierigkeiten in der Emotionsregulation erhöhen das Risiko einer psychischen Krankheit bereits im Jugendalter (z. B. Helmsen et al. 2012; Schramm et al. 2013; Zhao und Zhao 2015). Vor diesem Hintergrund erscheint die Erforschung von Emotionen und Prozessen der Emotionsregulation im Jugendalter höchst relevant. Fähigkeiten im Umgang mit negativen Emotionen sind zudem zentrale Elemente des so genannten Integrationsniveaus der psychischen Struktur (Arbeitskreis-OPD 1996, 2006; Rudolf 2002). Zusammenhänge zwischen dem Strukturniveau und dem Schweregrad psychischer Störungen sind sowohl im Erwachsenenbereich (Arbeitskreis-OPD 2006; Benecke et al. 2009; Zimmermann et al. 2012) als auch im Jugendalter (Benecke et al. 2011; Stefini et al. 2013) belegt, und erlauben Prognosen über den weiteren Verlauf einer psychischen Erkrankung bei Erwachsenen (Zimmermann et al. 2010).

\section{Der Verlauf psychischer Störung von Adoleszenz ins Erwachsenenalter}

Prognosen über die Entwicklung psychischer Störungen sind aufgrund der Komplexität der Einflussfaktoren nach wie vor sehr schwierig. Mittlerweile liegt eine Fülle von Studien vor, welche die Zusammenhänge zwischen unterschiedlichen Risikofaktoren und dem Vorliegen späterer psychischer Störungen untersuch- ten. Als Risikofaktoren wurden genetische, sozioökonomische Variablen, Merkmale der Eltern (siehe z. B. Farrington 2000; Fonagy et al. 2002; Herpertz-Dahlmann et al. 2003; Laucht et al. 1992; Petermann und Niebank 1999; Schulte-Markwort et al. 2001), sowie belastende Lebenserfahrungen (insbesondere Missbrauch, Misshandlung, Vernachlässigung usw.; Dubo et al. 1997; Egle et al. 2005; Gladstone et al. 1999; Golier et al. 2003; Grilo und Masheb 2002; Léonard et al. 2003; Zanarini et al. 1997) ausgemacht, bzw. Kombinationen aus diesen Faktoren (z. B. Bifulco et al. 2002; Carter et al. 2001; Joyce et al. 2003; Mulder et al. 1994).

Trotz der Aufhebung der Altersgrenze bei der Diagnose von Persönlichkeitsstörungen im Jugendalter entsprechend der Neuerungen im DSM-5, wird eine Diagnostizierbarkeit im klinischen Alltag nach wie vor intensiv diskutiert. In Zentrum stehen dabei vor allem die Verläufe und die Persistenz der Störungen (Krischer et al. 2006; Sevecke und Krischer 2011). Diesbezügliche Studien fanden, dass das Vorliegen einer adoleszenten Persönlichkeitsstörung die Wahrscheinlichkeit einer Persönlichkeitsstörung des gleichen Clusters im frühen Erwachsenenalter erhöht (Kasen et al. 1999). Dabei stellen komorbide Achse-I und Achse-II Störungen im Jugendalter ein besonders hohes Risiko für eine spätere psychische Krankheit dar (Crawford et al. 2008). Insgesamt sanken die Persistenzraten von Persönlichkeitsstörungen jedoch deutlich, wenn anstelle einzelner Merkmale lediglich vollständige Diagnosen von Persönlichkeitsstörungen in Betracht gezogen werden (vgl. auch bspw. Becker et al. 1999; Bernstein et al. 1993; Mattanah et al. 1995; Meijer et al. 1998). Andere Studien (z. B. Johnson et al. 2000, 2008) fanden dagegen einen signifikanten Rückgang des Vorliegens diagnostischer Merkmale von Persönlichkeitsstörungen, insbesondere zwischen dem 16. und 22. Lebensjahr (vgl. auch Lenzenweger 1997).

Insgesamt zeigen die Studien, dass das Vorliegen von psychischer Symptomatik in Kindheit und Jugend das Risiko psychischer Störungen zu späteren Zeitpunkten erhöht. Gleichzeitig zeigt sich aber auch eine starke Heterogenität der Verläufe mit Symptomwechseln, Remissionen und Neu-Manifestationen, die insbesondere individuelle Verlaufsprognosen erschweren. Diese starke Heterogenität der Verläufe, von Persistenzen bis Remissionen, Wechsel von Achse-I zu Achse-II und umgekehrt, teilweises Verschwinden und Wiederauftreten einzelner Symptomatiken etc., sprechen u. E. für die stärkere Fokussierung auf zugrundeliegende, basale Dimensionen, wie bspw. das Integrationsniveau der psychischen Struktur, oder Charakteristiken der Emotionsregulierung. Die jeweils aktuelle Symptomatik kann nur als temporäres Endprodukt der Entwicklung verstanden werden, sie kann wechseln oder „ruhen“; die basalen Variablen sind wahrscheinlich stabiler. Lehmkuhl (2006) schlägt zur Entwicklung differenzierter Behandlungsstrategien die Untersuchung der Frage, „wie sich verschiedene 
Gruppen hinsichtlich ihrer affektiven Symptomatik und aufgrund von Symptomprofilen identifizieren lassen" (S. 99) vor, um daraus entsprechende Konsequenzen für ein differenzielles therapeutisches Vorgehen abzuleiten.

\section{Emotionserleben und Emotionsregulation bei Jugendlichen mit psychischen Störungen}

Da ein Großteil unseres psychischen Geschehens in emotionale Prozesse eingebunden ist, und psychische Störungen immer auch als emotionale Störungen beschrieben werden können (Benecke 2014; Rudolf und Henningsen 2008), stellt die Erfassung des emotionalen Erlebens eine Möglichkeit dar, einen differenzierteren Blick auf zugrundeliegende Dimensionen psychischer Störungen im Jugendalter zu werfen. Studien dazu gibt es vor allem für psychisch erkrankte Erwachsene. Die Ergebnisse hierzu zeigen ein Vorherrschen negativer Emotionen und reduziertes positives Emotionserleben (z. B. Benecke 2006; Benecke et al. 2008; Highfield et al. 2010; Kämmerer 2010; Kashdan et al. 2013; Leising et al. 2004; Myin-Germeys et al. 2003; Yuan und Kring 2009).

Deutlich weniger empirische Untersuchungen liegen zum subjektiven Emotionserleben von Jugendlichen vor. In einer der wenigen Studien, die das subjektive Erleben von spezifischen Emotionen jenseits einer positiv-negativ-Dichotomie untersuchten, reagierten gewalttätige Jugendliche mit weniger subjektiv empfundener Schuld und Angst, aber mit mehr Freude und Erregung auf eine Gesichte, in der der Protagonist eine antisoziale Handlung ausgeübt hat, als nicht-gewalttätige Jugendliche (Cimbora und McIntosh 2003). Marsh et al. (2011) fanden bei Jugendlichen mit Persönlichkeitsaspekten der Psychopathie reduziertes subjektives Erleben von Angst. BorderlineSymptomatik stand bei jugendlichen Mädchen in Zusammenhang mit feindseliger Gereiztheit und Scham (Scott et al. 2015), während bei depressiven Jugendlichen eine Reduktion positiven Emotionen gefunden wurde (Fussner et al. 2015).

Ebenso wie verändertes Emotionserleben stehen überdauernde Defizite der Emotionsregulation in engem Bezug zu psychischer Erkrankung.

Prozesse der Emotionsregulierung wurden bei Jugendlichen mit psychischen Störungen vor allem in den letzten Jahren umfangreicher untersucht. Sharp et al. (2011) fanden bei Jugendlichen mit BorderlinePersönlichkeitsaspekten, dass Hypermentalisierung (Überinterpretation mentaler Zustände anderer) indikativ für Schwierigkeiten in der Emotionsregulation war. Kim et al. (2014) zeigten, dass eine sichere Bindung zu den Eltern (v. a. zum Vater) als Puffer für Borderline-Persönlichkeitsaspekte diente, indem sie positive Emotionsregulationsstrategien erhöhte. Maladaptive Emotionsregulationsstrategien standen in Zusammenhang mit Borderline-Symptomatik, da sie protektive Aspekte sicherer Bindung abschwächten.
Weiter stand die Schwere von Borderline-Persönlichkeitsaspekten in Zusammenhang mit Schwierigkeiten in der Emotionsregulation und dem Vermeiden von emotionalen Erfahrungen (bspw. in Kontakt bleiben mit unangenehmen Erinnerungen, Gedanken, Gefühlen, Drängen) (Schramm et al. 2013). Ein vergleichbares Ergebnis wurde bei alexithymen Jugendliche gefunden (Venta et al. 2012). Zusammengenommen klärten Impulsivität und emotionale Dysregulation mehr als ein Drittel der Varianz von Borderline-Merkmalen in einer nichtklinischen jugendlichen Stichprobe auf (Fossati et al. 2013). Schwierigkeiten in der Emotionsregulation stellten auch im Rahmen nichtsuizidaler Selbstverletzungen ein Kernproblem dar (In-Albon et al. 2015; Voon et al. 2014a, 2014b).

Zhao und Zhao (2015) fanden, dass vor allem die Unterdrückung von Emotionen als Regulationsstrategie mit erhöhter depressiver Symptomatik bei Jugendlichen einherging. Eine andere Studie zeigte: Je dysregulierter das emotionale Verhalten depressive Jugendlicher war, desto dysregulierter waren sie auch physiologisch (Crowell et al. 2014). Ein Überblick zu positiven Emotionen und Psychopathologie bei Jugendlichen von Gilbert (2012) fand bei Depressionen zudem Zusammenhänge zu Dysregulation von positiven Emotionen (bspw. Anhedonie und geringere Responsivität gegenüber positiver Stimuli). Der Vergleich von depressiven Jugendlichen, Jugendlichen mit Angststörung und Jugendlichen ohne Diagnose zeigte, dass sowohl depressive als auch Jugendliche mit Angststörung maladaptivere Emotionsregulationsstrategien anwendeten als gesunde Jugendliche. Die beiden klinischen Gruppen unterschieden sich jedoch nicht voneinander (Kullik und Petermann 2013).

Queens und Ehrenreich-May (2014) fanden bei Jugendlichen mit Angststörungen, dass eine komorbide Depression mit unterdrücktem Ausdruck negativer Emotionen, sowie unterdrücktem emotionalen Empfinden zusammenhing. Jugendliche mit sozialen Ängsten verwendeten weniger adaptive Strategien als nicht-ängstliche Jugendliche (Lange und Tröster 2014) und berichteten über geringere emotionale Klarheit, geringere Überzeugung die eigenen Emotionen bewältigen zu können, und stärkere Reaktivität bei negativen Emotionen (Mathews et al. 2014). Geringe Verfügbarkeit über effektive Emotionsregulationsstrategien und Mangel an emotionaler Klarheit war bei Mädchen prädiktiv für Ängste, bei Jungen war hingegen Inakzeptanz emotionaler Reaktionen ein Prädiktor (Bender et al. 2012).

Essstörungen standen bei Jugendlichen in Zusammenhang mit emotionalem Missbrauch und Schwierigkeiten in der Emotionsregulation. Zudem war dysfunktionale Emotionsregulation eine Mediatorvariable zwischen kindlichem emotionalen Missbrauch und Essstörungen im Erwachsenenalter (Mills et al. 2015), sowie ein partieller Mediator zwischen Bindungsangst bzw. bindungsvermeidendem Verhalten 
gegenüber der Mutter und Einschränkungen und Bedenken bezüglich des Essens (Van Durme et al. 2015).

Greuel et al. (2015) fanden bei psychisch kranken Jugendlichen geringere Werte auf allen Skalen adaptiver Emotionsregulierung als bei gesunden Jugendlichen.

\section{„Struktur" und psychische Störung}

„Struktur“ bezeichnet in der modernen psychodynamischen Literatur die Verfügbarkeit über basale psychische Funktionen, wie z. B. eine differenzierte Selbst- und Fremdwahrnehmung, die Fähigkeit zur Selbststeuerung/Impulskontrolle, oder Bindungssicherheit. Das Konzept psychischer Struktur überschneidet sich in vielen Bereichen mit dem Konzept der so genannten „Mentalisierung“ - von Fonagy et al. (2002) als "the capacity to envision mental states in self and others “ (S. 23) beschrieben. Zusammenhänge zwischen psychischen Störungen und Defiziten der Mentalisierung sind gut belegt (Bouchard et al. 2008; Fonagy et al. 1996, 2002); ebenso sind die Zusammenhänge zwischen dem „Strukturniveau“ der Operationalisierten Psychodynamischen Diagnostik (OPD; Arbeitskreis OPD 2006) und psychischen Störungen deutlich (Benecke et al. 2011; Zimmermann et al. 2012).

Vor allem in den letzten Jahren fand das Strukturniveau gemäß der Operationalisierten Psychodynamischen Diagnostik im Kindes- und Jugendalter (OPDKJ; Arbeitskreis OPD-KJ 2007) vermehrt Eingang in Studien mit psychisch kranken Jugendlichen. Interrater-Reliabilitäts- und Validitätsstudien des OPDKJ zeigten gute bis sehr gute Interrater-Reliabilität für die Gesamtstruktur und die strukturellen Subbereiche (Steuerung, Selbst- und Objekterleben, Kommunikative Fähigkeiten), sowie hochsignifikante Zusammenhänge aller Strukturbereiche mit gleichzeitig vorliegenden DSM-IV Diagnosen der Achse II (Benecke et al. 2011). Niedrigere Gesamtstruktur korrelierte zudem tendenziell mit höherer Symptombelastung (entsprechend des Global Severity Index des BSI) und mit Defiziten der Persönlichkeitsorganisation (entsprechend des IPO; v. a. in der Skala „primitive Abwehr“) (Benecke et al. 2011). Stefini et al. (2013) belegten zudem gute bis hervorragende Interrater-Reliabilität der Achsen „Konflikt“ und „Struktur“ bei einer Untersuchung von Jugendlichen und jungen Erwachsenen mit Bulimia nervosa. Die OPD-KJ-Strukturachse erzielte auch beim Einsatz im Rahmen klinischer Routine hinreichend gute Reliabilitäts- und Validitätswerte (Cropp et al. 2013; Weitkamp et al. 2013). Kiene (2007) fand, dass die Struktur-Dimension klar zwischen aggressiven und nicht-aggressiven Jugendlichen differenzierte. Seiffge-Krenke et al. (2013) fanden, dass Kinder und Jugendliche, die einer stationären Therapie zugewiesen wurden, ein signifikant schlechteres Strukturniveau aufwiesen als jene in ambulanter Therapie. Der gravierendste Unterschied wurde hierbei im Strukturbereich „Steuerung“ gefunden, woraus die AutorInnen schlossen, dass vor allem der Umgang mit negativen Emotionen im therapeutischen Rahmen stark gefördert werden müsse. Zur Beurteilung von Veränderungen der psychischen Struktur bei Kindern und Jugendlichen mit psychiatrischen Störungen wurde die OPD-KJ Achse „psychische Struktur“ zu Beginn eines stationären Aufenthalts, sowie 2 Jahre nach der Entlassung erhoben. Es zeigten sich eine Verbesserung der Gesamtstruktur, sowie Verbesserungen in den Strukturbereichen „Steuerung“ und „Kommunikative Fähigkeiten“, nicht jedoch für das „Selbst- und Objekterleben“ (Jelen-Mauboussin et al. 2013).

Entsprechend der Defizite in psychisch-struktureller Hinsicht, steht auch defizitäre Mentalisierungsfähigkeit mit psychischer Krankheit im Jugendalter in Verbindung. So wurde bei männlichen Jugendlichen, die erhöhte Tendenzen der Psychopathie und Aggressivität aufwiesen, geringere Mentalisierungsfähigkeit (gemessen als reflexive Kompetenz) festgestellt (Taubner et al. 2010). Jugendliche mit erhöhten Tendenzen von Psychopathie waren jedoch nur dann erhöht aggressiv, wenn sie über moderate bis geringe reflexive Kompetenzen verfügten, nicht jedoch bei guten reflexiven Fähigkeiten (Taubner et al. 2013). Jugendliche, die mit 12 Jahren andere Kinder schikanierten, oder von anderen schikaniert wurden, hatten bereits mit 5 Jahren geringere Fähigkeiten zu mentalisieren (gemessen als Theory of Mind; Shakoor et al. 2012). Auch bei Borderline-Persönlichkeitsaspekten zeigte sich: Mit zunehmender Borderline-Symptomatik nahmen die reflexiven Kompetenzen der Jugendlichen ab (z. B. Fossati et al. 2014; Ha et al. 2013; Sharp et al. 2011). Jugendliche mit bipolarer Störung lösten Theory-of-Mind-Aufgaben schlechter als gesunde Jugendliche; früher Krankheitsbeginn und manische Symptome wirkten sich hierbei zusätzlich negativ auf die Mentalisierungsfähigkeit aus (Schenkel et al. 2008).

Insgesamt lassen die vorliegenden Befunde zum Zusammenhang zwischen strukturellen Funktionen und psychischen Störungen vermuten, dass die strukturellen Dimensionen das Risiko zur Entwicklung einer schweren psychischen Störung im Übergang vom Jugend- ins Erwachsenenalter besonders gut vorhersagen. Dies bedeutet weiter, dass bei Vorliegen einer strukturellen Beeinträchtigung im Jugendalter (unabhängig von der aktuellen Symptomatik) gezielte Interventionsstrategien indiziert sind. Aufgrund des hohen Aufwands einer ausführlichen Strukturdiagnostik könnte ein schneller durchführbares „Struktur-Screening“ im klinischen Alltag eine wünschenswerte Erleichterung darstellen.

\section{Ziel der Studie}

Es soll geprüft werden, ob strukturelle Störungen im Jugendalter anhand eines „Risiko-Screenings“ durch zwei Selbsteinschätzungsinstrumente vorhergesagt 
Abb. 1 Verteilung der Strukturniveaus der klinisch auffälligen und unauffälligen Jugendlichen. (Klinisch auffällig zum Zeitpunkt der Untersuchung stationär in der Kinder-Jugend-Psychiatrie)

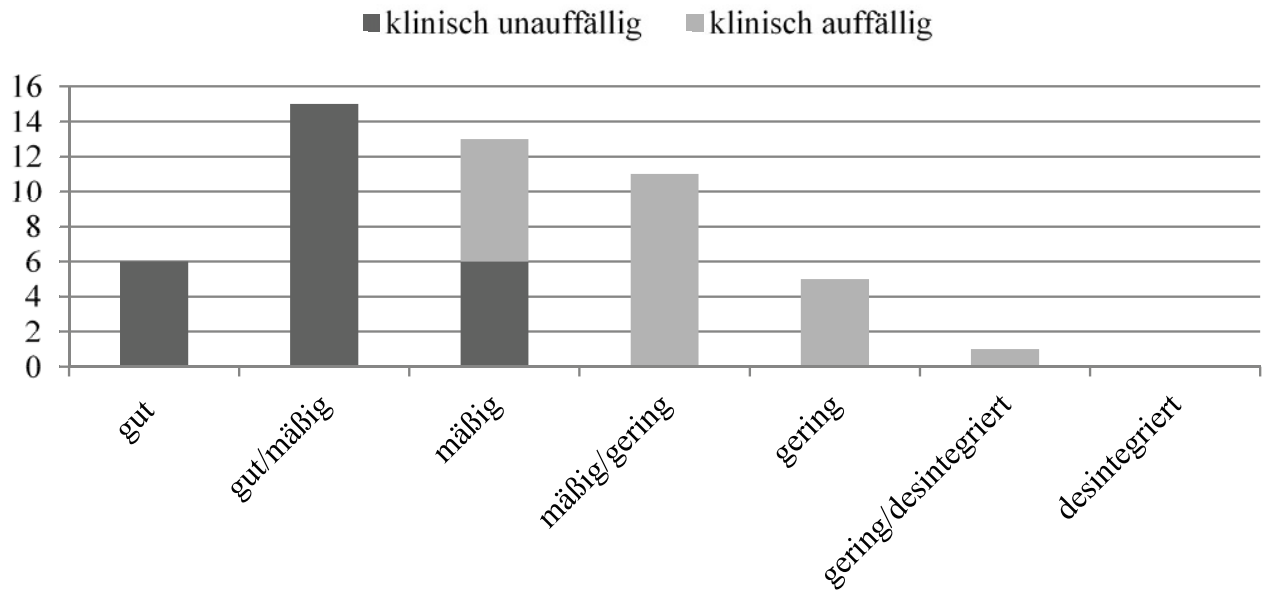

werden können. Die psychische Struktur der Jugendlichen wird anhand der Strukturachse des OPD-KJ (Arbeitskreis-OPD-KJ 2007) gemessen. Die beiden Selbsteinschätzungsverfahren zum Risiko-Screening erheben das emotionale Erleben und Charakteristiken der Emotionsregulation (gemessen mit dem EER; Benecke et al. 2008), sowie Merkmale der Persönlichkeitsorganisation (nach Kernberg 1984; Kernberg und Caligor 2005) der Jugendlichen (Identitätsdiffusion, primitive Abwehr, Realitätsprüfung; gemessen mit dem IPO-16, Zimmermann et al. 2013).

\section{Fragestellung und Methodik}

\section{Stichprobe}

Die Stichprobe für diesen Beitrag umfasst 51 Jugendliche zwischen 14 und 17 Jahren $(M=15,63$; $S D=0,89)$. Ein etwas größerer Teil der Stichprobe ist weiblich ( $n=28,54,9 \%$ ), 45,1 \% sind männliche Jugendliche ( $n=23)$. Neben stationären PatientInnen der Universitätsklinik für Kinder- und Jugendpsychiatrie Innsbruck ( $n=24$ ) wurden zwei Jugendliche über eine betreute Wohngruppe, und weitere 25 Jugendliche über Schulen und persönliche Kontakte rekrutiert. Von den 51 ProbandInnen erfüllen 18 (35,2\%) eine oder mehrere Diagnosen nach SKID-II (Fydrich et al. 1997), 32 (62,7\%) ProbandInnen haben keine SKIDII-Diagnose, bei einem Probanden wurde kein SKIDII durchgeführt. Die 27 Jugendlichen, die nicht PatientInnen der Kinder- und Jugendpsychiatrie waren, füllten den PHQ (Löwe et al. 2002) aus. Dieser Fragebogen deckt weitgehend die Achse-I-Störungen ab. Im Fall der Überschreitung der Cutoff-Werte des PHQ wurde weiterführend ein SKID-I-Interview durchgeführt. Es zeigte sich, dass diese 27 Jugendlichen als „klinisch unauffällig“ eingestuft werden können, da sie weder eine Achse-I- noch eine Achse-II-Störung aufweisen.

Mit allen Jugendlichen wurde ein OPD-KJ Interview (Arbeitskreis-OPD-KJ 2007) durchgeführt, zudem füllten sie eine umfangreiche Fragebogenbatterie aus. Im
Folgenden werden nur jene Instrumente beschrieben, die für den vorliegenden Beitrag relevant sind. Für einen umfassenden Überblick über die Studie siehe Benecke et al. (2011).

\section{Erhebungsinstrumente}

\section{OPD-KJ}

Die Operationalisierte Psychodynamische Diagnostik für Kinder und Jugendliche (Arbeitskreis OPDKJ 2007) ist ein System zur Einschätzung klinisch relevanter Merkmale auf den Achsen Krankheitserleben, Konflikt, Beziehung und Struktur. Je nach Altersstufe sind unterschiedliche Quellen für die Ratings zu empfehlen. In der Altersstufe 3 (ab 13 Jahren) können, wie bei der OPD für Erwachsene (OPD-E; Arbeitskreis OPD 1996, 2006), halbstrukturierte Interviews durchgeführt werden. Die Strukturachse des OPD-KJ beschreibt auf drei Dimensionen (Steuerung; Selbst- und Objekterleben; Kommunikative Fähigkeiten) sowie einer Gesamteinschätzung jeweils vier Integrationsniveaus der psychischen Struktur (1: gut integriert, 2: mäßig integriert, 3: gering integriert, 4: desintegriert). Die in der vorliegenden Studie verwendeten Interviews wurden von sieben verschiedenen InterviewerInnen geführt und dauerten je ca. $90 \mathrm{Mi}-$ nuten.

Das Rating erfolgte feinkategorial, d.h. zu den vier Integrationsstufen wurden noch jeweils drei Zwischenstufen geratet $(1 / 1,5 / 2 / 2,5 / 3 / 3,5 / 4$; analog zur OPD-E). Das Strukturrating im Forschungsprojekt wich in einigen Punkten vom OPD-KJ Manual von 2007 ab. Der Strukturaspekt „Selbsterleben“ enthielt in seiner manualisierten Formulierung drei verschiedene Aspekte: Selbstwahrnehmung, Affektdifferenzierung und Identität, die aber alle unter „Selbsterleben“ subsummiert wurden. Im vorliegenden Forschungsprojekt wurde dieser Strukturaspekt weiter unterteilt, indem zwischen „Selbst/Affektdifferenzierung“ und „Identität“ unterschieden wurde; im Gesamtrating wurde zwischen Selbsterleben und Objekterleben unterschieden, da dies bei vielen ProbandInnen deutlich 
auseinander fiel (siehe Benecke et al. 2011). Im OPDKJ-2 (Arbeitskreis-OPD-KJ 2013) wurden diese Abweichungen in das Manual übernommen.

Die OPD-KJ Ratings des Strukturniveaus dieser Jugendlichen wurden jeweils vorab im Einzel-Rating durchgeführt, und anschließend ein in der Gruppe erzielter Konsens getroffen. Die Gruppe der zertifizierten RaterInnen blieb konstant, wobei aufgrund allfälliger Abwesenheit einzelner RaterInnen bei jedem Jugendlichen eine unterschiedliche Anzahl von Einzelratings vorliegt (siehe Benecke et al. 2011).

Das siebenstufige feinkategoriale Rating des Strukturniveaus nach OPD-KJ verteilt sich wie folgt: Gute Integration bei 6 (11,8\%) ProbandInnen, zwischen gut und mäßig bei 15 (29,4\%), mäßige Integration bei 13 (25,5\%), zwischen mäßig und gering bei 11 (21,6\%), geringe Integration bei $5(9,8)$ ProbandInnen, 1 Proband $(2,0 \%)$ wurde als zwischen gering und desintegriert geratet, kein/e ProbandIn fiel in die Strukturstufe „desintegriert“. Abb. 1 zeigt die Verteilung der klinisch unauffälligen und der klinisch auffälligen Jugendlichen in den feinkategorialen Strukturstufen.

\section{Fragebögen}

\section{Fragebogen zur Erfassung von Emotionserleben und} Emotionsregulation (EER)

Der Fragebogen zur Erfassung von Emotionserleben und Emotionsregulation (EER; Benecke et al. 2008) ist ein Selbsteinschätzungsinstrument. Der EER erfasst zum einen das subjektive Emotionserleben, zum anderen werden Strategien der Emotionsregulation für eine als schwierig empfundene Emotion erhoben. Der Erlebensteil des EER umfasst 20 Skalen; angegeben wird die Intensität des Erlebens verschiedener Emotionen in den letzten 7 Tagen auf einer 7-stufigen Antwortskala. Der Regulationsteil besteht aus 15 Skalen. Zur Bearbeitung wählt der/die ProbandIn eine subjektiv schwierige Emotion aus dem Erlebnisteil des EER aus. Anschließend werden wiederum auf einer 7-stufigen Antwortskala Reaktionen auf bzw. der Umgang mit dieser Emotion angegeben. Die Skalen des Regulationsteils sind Verwirrung, Überforderung, Rückzug, Dissoziation, Körper-Ausdruck, Körper nutzen, Reflexion, soziale Unterstützung, Perspektivenübernahme, Externalisierung, Musterreflexion, Ablenkung, Black-out, Spontanität und Sich verlieren, und zeigen je unterschiedliche Zusammenhänge mit der Schwere psychischer Symptomatik (siehe: Benecke et al. 2008). Die 20 Erlebensskalen konvergieren in drei Faktoren 2. Ordnung: passiv-negative, aktiv-negative, sowie positive Emotionen (siehe Tab. 1 und 2); die Regulierungsskalen lassen sich zu vier übergeordneten Skalen zusammenfassen (siehe Tab. 1 und 3). Die teststatistischen Kennwerte des EER zeigen eine gute interne Konsistenz und hohe Zusammenhänge zu psychischer Symptomatik bei Erwachsenen (Benecke et al. 2008). Für den Jugendbereich liegt bisher noch keine entsprechende Überprüfung vor.
Inventar zur Erfassung der Persönlichkeitsorganisation (IPO-16)

Die Kurzform des Inventory of Personality Organization (Zimmermann et al. 2013, 2015) erfasst das Ausmaß an Persönlichkeitsbeeinträchtigung gemäß dem Modell der Persönlichkeitsorganisation von Kernberg (1984; Kernberg und Caligor 2005) anhand der Bereiche Identität, Abwehr und Realitätsprüfung. Das IPO16 verfügt über sehr gute Testgütekriterien. Zudem liegen bevölkerungsrepräsentative Normen und Cut-OffWerte für das Vorliegen einer Persönlichkeitsstörung gemäß DSM-IV, sowie einer struktureller Störung gemäß OPD vor (Zimmermann et al. 2015). Für den Jugendbereich gibt es hierzu noch keine Ergebnisse.

\section{Ergebnisse}

\section{Zusammenhänge der OPD-KJ-Struktur-Ratings mit} EER und IPO-16

Zur Ermittlung der Zusammenhänge der OPD-Struktur-Ratings mit den Fragebögen EER und IPO-16 wurden die siebenstufigen OPD-Struktur-Feinkategorien der Gesamtskala und der vier Unterskalen verwendet. Im EER wurden die Items zu ihren Subskalen sowie zu den übergeordneten Skalen per Mittelwertbildung zusammengefasst. Fehlte bei einer Person ein einzelner Item-Wert auf einer Subskala, so wurde dieser durch den Mittelwert der übrigen Items der Subskala ersetzt. Ebenso wurde beim IPO-16 vorgegangen. Als Korrelationsmaß wurde wegen der Ordinalskalierung der Strukturachse und der vorliegenden Rangbindungen Kendall's Tau verwendet.

Tab. 2 zeigt die Zusammenhänge mit dem Erlebensteil des EER. Es zeigt sich ein positiver Zusammenhang der Strukturgesamtskala mit dem Erleben passiv-negativer Emotionen. Dies gilt vor allem für die Subskalen Angst, Vernichtungsangst, Leblosigkeit und Einsamkeit. Personen, die diese Emotionen häufiger erleben, sind strukturell beeinträchtigter. Weitere, in diesem Fall negative, Zusammenhänge ergeben sich mit dem Erleben positiver Emotionen in den Subskalen Freude und Interesse. Personen, die mehr Freude und Interesse erleben, sind demnach weniger strukturell beeinträchtigt. Das Erleben von aktiv-negativen Emotionen hängt hingegen nicht mit dem Strukturniveau zusammen.

Im Regulationsteil des EER lassen sich nur wenige signifikante Korrelationen mit dem Strukturniveau finden (siehe Tab. 3). Ein deutlich positiver Zusammenhang ergibt sich mit der Emotionsregulationsstrategie Blackout; ein negativer Zusammenhang zeigt sich mit der Strategie Empathie. Höhere strukturelle Beeinträchtigung geht also mit häufigerem Anwenden der Strategie Blackout und seltenerer Anwendung der Strategie Empathie einher.

Beim IPO-16 zeigt sich nur mit der Subskala primitive Abwehr ein signifikant positiver Zusammenhang (siehe Tab. 4). 


\section{originalarbeit}

Tab. 1 Deskriptive Statistiken der OPD-Strukturratings sowie der Skalen des EER und IPO-16 ( $N=51)$

\begin{tabular}{|c|c|c|c|c|}
\hline & Min & Max & $M$ & SD \\
\hline \multicolumn{5}{|l|}{ OPD Strukturratings } \\
\hline Struktur gesamt & 1,00 & 3,50 & 1,97 & 0,63 \\
\hline Steuerung & 1,00 & 3,50 & 2,04 & 0,71 \\
\hline Selbsterleben & 1,00 & 3,50 & 1,88 & 0,62 \\
\hline Objekterleben & 1,00 & 3,50 & 2,01 & 0,68 \\
\hline Kommunikation & 1,00 & 3,50 & 1,89 & 0,57 \\
\hline \multicolumn{5}{|l|}{ EER Erlebensteil } \\
\hline Passiv-negativ & 0,08 & 4,75 & 1,50 & 1,12 \\
\hline Angst & 0,00 & 6,00 & 1,27 & 1,41 \\
\hline Vernichtungsangst & 0,00 & 5,00 & 1,16 & 1,25 \\
\hline Scham & 0,00 & 3,33 & 1,30 & 1,02 \\
\hline Diffuse Angst & 0,00 & 5,67 & 1,86 & 1,36 \\
\hline Trauer & 0,00 & 5,67 & 2,41 & 1,58 \\
\hline Leblosigkeit & 0,00 & 5,67 & 1,39 & 1,69 \\
\hline Einsamkeit & 0,00 & 5,67 & 1,44 & 1,68 \\
\hline Schuld & 0,00 & 5,33 & 1,20 & 1,04 \\
\hline Aktiv-negativ & 0,00 & 4,83 & 1,74 & 1,01 \\
\hline Ekel & 0,00 & 5,67 & 1,30 & 1,48 \\
\hline Unbeherrschtheit & 0,00 & 6,00 & 1,70 & 1,27 \\
\hline Reizbarkeit & 0,00 & 6,00 & 2,16 & 1,55 \\
\hline Wut & 0,00 & 6,00 & 2,64 & 1,58 \\
\hline Impulsivität & 0,00 & 5,67 & 1,92 & 1,20 \\
\hline Hemmungslosigkeit & 0,00 & 5,00 & 1,62 & 1,20 \\
\hline Verachtung & 0,00 & 3,67 & 1,22 & 1,08 \\
\hline Neid, Eifersucht & 0,00 & 6,00 & 1,34 & 1,39 \\
\hline Positiv & 0,25 & 4,58 & 2,86 & 0,95 \\
\hline Freude & 0,00 & 5,67 & 3,74 & 1,36 \\
\hline Liebe, Zärtlichkeit & 0,00 & 6,00 & 2,83 & 1,61 \\
\hline Überraschung & 0,00 & 4,67 & 2,10 & 1,31 \\
\hline Interesse & 0,00 & 4,67 & 2,76 & 1,20 \\
\hline \multicolumn{5}{|l|}{ Regulationsteil } \\
\hline Dissoziation & 0,22 & 5,22 & 2,71 & 1,30 \\
\hline Verwirrung & 0,00 & 5,67 & 2,92 & 1,64 \\
\hline Belastung, Überforderung & 0,00 & 6,00 & 3,08 & 1,87 \\
\hline Rückzug, Lähmung & 0,00 & 6,00 & 3,10 & 1,86 \\
\hline Dissoziation & 0,00 & 6,00 & 2,61 & 1,78 \\
\hline Blackout & 0,00 & 4,00 & 1,44 & 1,18 \\
\hline Sich verlieren & 0,00 & 6,00 & 3,08 & 1,55 \\
\hline Reflexion & 0,00 & 5,08 & 2,77 & 1,25 \\
\hline Körperwahrnehmung (Nutzen) & 0,00 & 5,67 & 2,28 & 1,66 \\
\hline Reflexion & 0,00 & 6,00 & 2,89 & 1,46 \\
\hline Soziale Unterstützung & 0,00 & 6,00 & 3,02 & 2,15 \\
\hline Empathie & 0,00 & 6,00 & 2,92 & 1,71 \\
\hline \multicolumn{5}{|l|}{ IP0-16 } \\
\hline Gesamtwert & 1,06 & 4,31 & 2,26 & 0,81 \\
\hline Identitätsdiffusion & 1,00 & 4,33 & 2,36 & 0,88 \\
\hline Primitive Abwehr & 1,00 & 4,20 & 2,49 & 0,96 \\
\hline Realitätsprüfung & 1,00 & 5,00 & 1,92 & 0,95 \\
\hline
\end{tabular}


Tab. 2 Korrelation der OPD-Struktur-Skalen mit den Emotionserlebensskalen des EER $(N=51)$

\begin{tabular}{|c|c|c|c|c|c|}
\hline Skalen zum Emotionserleben & $\begin{array}{l}\text { Struktur } \\
\text { gesamt }\end{array}$ & Steuerung & Selbsterleben & Objekterleben & Kommunikation \\
\hline Passiv-negativ & $0,23^{*}$ & $0,22^{*}$ & 0,19 & $0,22^{*}$ & 0,18 \\
\hline Angst & $0,24^{*}$ & 0,21 & 0,18 & $0,22^{*}$ & 0,21 \\
\hline Vernichtungsangst & $0,24^{*}$ & $0,27^{*}$ & $0,23^{*}$ & $0,23^{*}$ & 0,17 \\
\hline Scham & $-0,01$ & $-0,04$ & $-0,04$ & $-0,02$ & $-0,05$ \\
\hline Diffuse Angst & 0,11 & 0,10 & 0,10 & 0,13 & 0,07 \\
\hline Trauer & 0,19 & $0,22^{*}$ & 0,16 & 0,15 & 0,12 \\
\hline Leblosigkeit & $0,30^{* *}$ & $0,28^{*}$ & $0,24^{*}$ & $0,26^{*}$ & $0,24^{*}$ \\
\hline Einsamkeit & $0,35^{\star *}$ & $0,34^{* *}$ & $0,28^{*}$ & $0,30^{* *}$ & $0,30^{* *}$ \\
\hline Schuld & 0,02 & $-0,01$ & $-0,01$ & 0,03 & 0,06 \\
\hline Aktiv-negativ & $-0,02$ & $-0,07$ & 0,03 & 0,04 & $-0,02$ \\
\hline Ekel & 0,14 & 0,10 & 0,15 & 0,12 & 0,13 \\
\hline Unbeherrschtheit & $-0,11$ & $-0,14$ & $-0,04$ & $-0,08$ & $-0,10$ \\
\hline Reizbarkeit & $-0,09$ & $-0,13$ & $-0,02$ & 0,00 & $-0,11$ \\
\hline Wut & 0,13 & 0,10 & 0,19 & 0,19 & 0,11 \\
\hline Impulsivität & $-0,11$ & $-0,10$ & $-0,05$ & $-0,08$ & $-0,09$ \\
\hline Hemmungslosigkeit & $-0,10$ & $-0,08$ & $-0,06$ & $-0,08$ & $-0,09$ \\
\hline Verachtung & 0,15 & 0,12 & 0,15 & 0,16 & 0,11 \\
\hline Neid, Eifersucht & $-0,21$ & $-0,26^{*}$ & $-0,21$ & $-0,17$ & $-0,16$ \\
\hline Positiv & $-0,26^{*}$ & $-0,21^{*}$ & $-0,18$ & $-0,17$ & $-0,15$ \\
\hline Freude & $-0,29^{\star *}$ & $-0,28^{* \star}$ & $-0,23^{*}$ & $-0,21$ & $-0,20$ \\
\hline Liebe, Zärtlichkeit & $-0,10$ & $-0,09$ & $-0,05$ & 0,00 & 0,00 \\
\hline Überraschung & $-0,15$ & $-0,11$ & $-0,13$ & $-0,14$ & $-0,08$ \\
\hline Interesse & $-0,22^{*}$ & $-0,17$ & $-0,18$ & $-0,19$ & $-0,18$ \\
\hline
\end{tabular}

Tab. 3 Korrelation der OPD-Struktur-Skalen mit den Emotionsregulationsskalen des EER $(N=51)$

\begin{tabular}{|c|c|c|c|c|c|}
\hline Skalen zur Emotionsregulation & $\begin{array}{l}\text { Struktur } \\
\text { gesamt }\end{array}$ & Steuerung & Selbsterleben & Objekterleben & Kommunikation \\
\hline Dissoziation & 0,14 & 0,16 & 0,08 & 0,14 & 0,05 \\
\hline Verwirrung & 0,02 & 0,02 & $-0,03$ & 0,03 & $-0,05$ \\
\hline Belastung, Überford & 0,06 & 0,08 & $-0,02$ & 0,07 & $-0,01$ \\
\hline Rückzug, Lähmung & $<0,00$ & 0,05 & $-0,08$ & $-0,03$ & $-0,08$ \\
\hline Dissoziation & 0,15 & 0,18 & 0,14 & 0,20 & 0,07 \\
\hline Blackout & $0,26^{*}$ & $0,25^{\star}$ & $0,23^{*}$ & $0,23^{*}$ & 0,18 \\
\hline Sich verlieren & 0,17 & 0,19 & 0,14 & 0,16 & 0,14 \\
\hline Reflexion & $-0,25^{*}$ & $-0,20$ & $-0,20$ & $-0,17$ & $-0,20$ \\
\hline Körperwahrnehmung & $-0,10$ & $-0,10$ & $-0,09$ & $-0,07$ & $-0,10$ \\
\hline Reflexion & $-0,11$ & $-0,11$ & $-0,09$ & $-0,09$ & $-0,07$ \\
\hline Soz. Unterstützung & $-0,19$ & $-0,16$ & $-0,15$ & $-0,15$ & $-0,17$ \\
\hline Empathie & $-0,27^{*}$ & $-0,19$ & $-0,22^{*}$ & $-0,22^{*}$ & $-0,20$ \\
\hline
\end{tabular}

Vorhersage einer strukturellen Beeinträchtigung durch EER und IPO-16

Zur Vorhersage einer strukturellen Beeinträchtigung wurde die Gesamtstrukturskala dichotomisiert. Als strukturell beeinträchtigt gelten hiernach Personen, die ein Gesamtstrukturniveau von 2,5 oder niedriger haben $(n=17)$. Personen mit einem mäßigen bis guten Strukturniveau (2 und höher) bilden die wenig beeinträchtigte Gruppe $(n=34)$.

Die Subskalen des EER und IPO-16, bei denen ein Zusammenhang mit dem Strukturniveau gefunden wurde, wurden als Prädiktoren ausgewählt. Hierbei wurden Subskalen des Erlebensteils des EER, die zu einer Überkategorie (Erleben passiv-negativer beziehungsweise positiver Emotionen) gehörten, per Mittelwertbildung zusammengefasst. So bildeten die 
Tab. 4 Korrelation der OPD-Struktur-Skalen mit den Skalen des IPO-16 $(N=51)$

\begin{tabular}{|c|c|c|c|c|c|}
\hline Skalen des IP0-16 & $\begin{array}{l}\text { Struktur } \\
\text { gesamt }\end{array}$ & Steuerung & Selbsterleben & Objekterleben & Kommunikation \\
\hline Gesamtwert & 0,19 & 0,19 & 0,18 & 0,18 & 0,14 \\
\hline Identitätsdiffusion & 0,15 & 0,17 & 0,14 & 0,12 & 0,08 \\
\hline Primitive Abwehr & $0,24^{*}$ & $0,25^{\star}$ & $0,27^{\star}$ & $0,24^{*}$ & 0,16 \\
\hline Realitätsprüfung & 0,09 & 0,06 & 0,04 & 0,09 & 0,09 \\
\hline
\end{tabular}

Tab. 5 Univariate logistische Regressionen zur Vorhersage der Struktur-Gruppenzugehörigkeit ( $N=51)$

\begin{tabular}{|c|c|c|c|c|c|c|}
\hline & $B$ & Std.Fehler & Wald & $d f$ & $p$ & $\operatorname{Exp}(B)$ \\
\hline Erleben von Freude, Interesse (Mittel) & $-0,98$ & 0,33 & 8,81 & 1 & 0,003 & 0,37 \\
\hline Konstante & 2,34 & 1,04 & 5,07 & 1 & 0,024 & \\
\hline $\begin{array}{l}\text { Erleben von Angst, Vernichtungsangst, Leblosig-, Einsamkeit } \\
\text { (Mittel) }\end{array}$ & 0,58 & 0,24 & 6,11 & 1 & 0,013 & 1,79 \\
\hline Konstante & $-1,53$ & 0,47 & 10,51 & 1 & 0,001 & \\
\hline Regulationsstrategie Empathie & $-0,36$ & 0,19 & 3,53 & 1 & 0,060 & 0,70 \\
\hline Konstante & 0,31 & 0,59 & 0,27 & 1 & 0,605 & \\
\hline Regulationsstrategie Blackout & 0,74 & 0,29 & 6,64 & 1 & 0,010 & 2,10 \\
\hline Konstante & $-1,86$ & 0,58 & 10,25 & 1 & 0,001 & \\
\hline IP0-16 primitive Abwehr & 0,70 & 0,33 & 4,56 & 1 & 0,033 & 2,02 \\
\hline Konstante & $-2,51$ & 0,93 & 7,27 & 1 & 0,007 & \\
\hline
\end{tabular}

Tab. 6 Multivariate logistische Regressionen zur Vorhersage der Struktur-Gruppenzugehörigkeit $(N=51)$

\begin{tabular}{|c|c|c|c|c|c|c|}
\hline & $B$ & Std.Fehler & Wald & $d f$ & $p$ & $\operatorname{Exp}(B)$ \\
\hline \multicolumn{7}{|l|}{ Modell 1: 4 Prädiktoren } \\
\hline Erleben von Freude, Interesse (Mittel) & $-0,81$ & 0,40 & 4,06 & 1 & 0,044 & 0,44 \\
\hline $\begin{array}{l}\text { Erleben von Angst, Vernichtungsangst, Leblosig-, Einsamkeit } \\
\text { (Mittel) }\end{array}$ & $-0,07$ & 0,34 & 0,04 & 1 & 0,834 & 0,93 \\
\hline Regulationsstrategie Blackout & 0,50 & 0,34 & 2,14 & 1 & 0,143 & 1,65 \\
\hline IP0-16 primitive Abwehr & 0,28 & 0,43 & 0,43 & 1 & 0,514 & 1,32 \\
\hline Konstante & 0,41 & 1,81 & 0,05 & 1 & 0,819 & \\
\hline \multicolumn{7}{|l|}{ Modell 2: 2 Prädiktoren } \\
\hline Erleben von Freude, Interesse (Mittel) & $-0,83$ & 0,35 & 5,81 & 1 & 0,016 & 0,43 \\
\hline Regulationsstrategie Blackout & 0,53 & 0,31 & 2,98 & 1 & 0,084 & 1,70 \\
\hline Konstante & 1,04 & 1,27 & 0,68 & 1 & 0,410 & \\
\hline
\end{tabular}

Subskalen Angst, Vernichtungsangst, Leblosigkeit und Einsamkeit einen gemeinsamen Prädiktor der passivnegativen Emotionen; ebenso bildeten Freude und Interesse einen gemeinsamen Prädiktor der positiven Emotionen. Bei den Skalen des Regulationsteils korrelierte jeweils nur eine Subskala einer Überkategorie mit dem Strukturniveau, weshalb diese einzeln als Prädiktoren verwendet wurden (Blackout und Empathie). Gleiches gilt für die Skala primitive Abwehr des IPO-16.

Um die grundsätzliche Vorhersagekraft der einzelnen Prädiktoren zu ermitteln, wurden zunächst univariate logistische Regressionen berechnet, bei denen jeweils nur ein Prädiktor verwendet wurde, um die Gruppenzugehörigkeit der strukturellen Beeinträchtigung vorherzusagen. Hierbei zeigt sich, dass alle Variablen außer der Emotionsregulationsstrategie Empathie signifikante Prädiktoren für die Gruppenzuge- hörigkeit sind (siehe Tab. 5). Die Strategie Empathie wurde deshalb in den weiteren Analysen nicht berücksichtigt.

In einem zweiten Schritt wurde eine multivariate logistische Regression berechnet, bei der die verbleibenden vier Prädiktoren eingeschlossen wurden. Der Omnibustest weist das Modell insgesamt als signifikant besser als das Basismodell ohne Prädiktoren aus, $\chi(4)=14,90 ; p<0,01$. Die Varianzaufklärung nach Nagelkerke's $R^{2}$ beträgt 35,2\%. Die korrekte Gruppenzuweisung steigt von $66,7 \%$ im Basismodell auf 70,6\%. Allerdings zeigt sich, dass die Prädiktoren primitive Abwehr des IPO-16, sowie die zusammengefassten Skalen der passiv-negativen Emotionen weitab von Signifikanz als Einzelprädiktor liegen (siehe Tab. 6, Modell 1).

Daher wurde die Berechnung der logistischen Regression mit einer schrittweisen, rückwärts gerichte- 
ten Ausschlussstrategie wiederholt. Diese weist ein Modell mit den Prädiktoren der zusammengefassten Skalen der positiven Emotionen und der Emotionsregulationsstrategie Blackout als das geeignetste Modell aus. Tab. 6 (Modell 2) gibt einen Überblick über dieses Modell. Der Omnibustest zeigt, dass das Modell besser als das Basismodell ist, $\chi(4)=14,47 ; p=0,001$. Allerdings ist die Emotionsregulationsstrategie Blackout nur ein marginal signifikanter Prädiktor. Die Varianzaufklärung nach Nagelkerke's $R^{2}$ beträgt $34,2 \%$ und die korrekte Gruppenzuweisung steigt von $66,7 \%$ im Basismodell auf 76,5 \%. Hierbei ist zu erwähnen, dass eine korrekte Zuweisung in die Gruppe der strukturell weniger beeinträchtigen Patienten in 85,3\% erfolgt, in die Gruppe der strukturell beeinträchtigten Patienten in 58,8\%. Mittels Screening durch diese Subskalen des EER wird eine strukturelle Beeinträchtigung also in knapp $60 \%$ der Fälle erkannt.

\section{Zusammenfassung und Diskussion}

Eine ausführliche Diagnostik im kinder- und jugendpsychiatrischen Bereich sollte auch das strukturelle Niveau, beispielsweise gemäß OPD-KJ, umfassen. Vor allem hinsichtlich der wichtigsten jugend-psychiatrischen Differenzialdiagnose Adoleszentenkrise (Sevecke und Krischer 2011) ist der Einbezug psychischstruktureller Merkmale in die Diagnostik von erheblicher Bedeutung. Ähnlich dem Konzept der strukturellen Fähigkeiten, die mit der OPD-KJ-2 (Arbeitskreis OPD-KJ 2013) nun schon in seiner zweiten Auflage erfasst werden können, wurden im DSM-5 dimensionale Kriterien einer Persönlichkeitsstörung in Form der Levels of Personality Functioning aufgenommen. Ein entsprechender Vorschlag für die Jugendpsychiatrie findet sich auch bei Sevecke et al. (2014), die entsprechend der Neuerungen im DSM-5 das dort beschriebene dimensional-kategoriale Hybridmodell als Grundlage für das Verständnis von Persönlichkeitspathologie und Diagnostik im Jugendbereich nahelegen. Aufbauend auf diesem Modell ist eine Diagnose von Persönlichkeitsstörungen im Jugendalter möglich.

Die Strukturdiagnostik setzt jedoch eine Schulung voraus und ist zudem zeitintensiv. Die vorliegende Studie stellt den Versuch dar, ein schneller durchführbares „Risiko-Screening“ mittels zweier Selbstbeschreibungsinstrumenten zu prüfen, um aufbauend auf den Ergebnissen dieser Instrumente entscheiden zu können, ob es sich um strukturelle Defizite handelt, um ggf. anschließend eine ausführliche Diagnostik und Struktureinschätzung nach OPD-KJ durchzuführen.

Als potenzielle Prädiktoren wurden ein Fragebogen zur Erfassung von emotionalem Erleben und Emotionsregulation (EER), sowie eine Kurzform des Fragebogens zur Erfassung des Persönlichkeitsorganisationsniveaus (IPO-16) gewählt. Der IPO-16 hat im Erwachsenenbereich seine Tauglichkeit als StrukturScreening-Instrument bereits gezeigt (Zimmermann et al. 2015), ebenso sind die Bereiche Emotionserleben und Emotionsregulation theoretisch und empirisch eng mit der Schwere psychischer Beeinträchtigung im Erwachsenenbereich verknüpft (Benecke et al. 2008; Benecke 2014). Obwohl für den Jugendbereich keine Validierungen vorliegen, lässt sich annehmen, dass diese Instrumente auch gute Voraussetzungen für den Einsatz als Struktur-Screening bei Jugendlichen mitbringen könnten.

Die Prüfung der korrelativen Zusammenhänge zwischen den Fragebögen-Skalen und dem OPD-KJStrukturrating erbrachte folgende Ergebnisse: Jugendliche mit stärkerer struktureller Beeinträchtigung erleben passiv-negative Emotionen (insbesondere Angst, Vernichtungsangst, Leblosigkeit und Einsamkeit) signifikant stärker, und sie erleben positive Emotionen, vor allem Freude und Interesse, signifikant weniger. Zwischen dem Erleben von aktiv-negativen Emotionen, also allen Formen „aggressiver“ Emotionen, und dem Strukturniveau fanden sich keine signifikanten Zusammenhänge. Offensichtlich unterscheiden sich strukturell beeinträchtige und besser strukturierte Jugendliche nicht im Erleben der Spielarten aggressiver Affekte. Dies macht insofern Sinn, dass die Aktivierung von Aggressionen ein für diese Lebensphase normaler Prozess ist, sei es in Form von Wutausbrüchen, Aversion oder Verachtung gegenüber allem Etablierten. Auch Reizbarkeit und Impulsivität ist in diesem Alter offensichtlich kein Kennzeichen psychischer Beeinträchtigung. Auch, dass das Erleben von Scham und Schuld - bei Erwachsenen hoch korrelativ mit psychischer Belastung (Benecke et al. 2008) - bei Jugendlichen nicht mit dem Strukturniveau zusammenhängt, lässt sich durch die spezifische Lebensphase, in der insbesondere Scham auch bei psychisch Gesunden eine große Rolle spielt, gut erklären.

Im Gegensatz zu Erwachsenen (Benecke et al. 2008) korrelierten bei den Jugendlichen nur sehr wenige der Regulationsskalen des EER, nämlich Empathie und Blackout, mit dem Strukturniveau. Dies ist durch die Tatsache erklärbar, dass Jugendlichen allgemein mehr Schwierigkeiten in der Emotionsregulation zugesprochen werden (z. B. Steinberg 2005), sodass im vorliegenden Fall die Selbstangaben zur Emotionsregulation nicht systematisch mit struktureller Beeinträchtigung zusammenhingen. Die signifikanten Zusammenhänge zwischen höherer Empathie und besserer Struktur, sowie zwischen Blackout und schlechterer Struktur, sprechen dafür, dass diese beiden Reaktionsweisen auf subjektiv „schwierige Gefühle“ gewissermaßen von der normalen pubertären Fluktuation ausgenommen sind. Die Fähigkeit, im Angesicht subjektiv „schwieriger Gefühle“ zu verstehen, was andere denken oder fühlen, scheinen Jugendliche mit struktureller Beeinträchtigung nicht zur Verfügung zu haben; sie reagieren hingegen häufig mit einem Blackout, d. h. sie können z. B. ihren Körper nicht mehr wahrnehmen, sich später nicht mehr erinnern, was sich ereignet hat, etc. In der Mentali- 
sierungstheorie wird dies dahingehend erklärt, dass in Situationen mit hoher emotionaler Anspannung und psychosozialem Stress Individuen ab einem bestimmten Grad an Belastung nicht mehr auf die sonst vorhandenen Mentalisierungsfähigkeiten zurückgreifen können (Taubner und Sevecke 2015). Bei Jugendlichen mit geringerer struktureller Integration scheint dieser Punkt sehr viel früher erreicht $\mathrm{zu}$ sein, als bei besser strukturierten Jugendlichen.

Von den drei Skalen des IPO-16 korrelierte nur die Skala primitive Abwehr mit dem Strukturniveau gemäß OPD-KJ. Weder Identitätsdiffusion noch eingeschränkte Realitätsprüfung zeigten einen Zusammenhang mit dem Strukturniveau, ebenso wenig die Gesamtskala des IPO-16. Dies ist einerseits erstaunlich, da die drei Sub-Skalen bei Erwachsenen sehr hoch korrelieren und keine inkrementelle Validität besitzen, weshalb üblicherweise nur die Gesamtskala verwendet wird (Zimmermann et al. 2013, 2015). Kritisch anzumerken ist, dass einerseits die Normierung des IPO an einer erwachsenen Stichprobe durchgeführt wurde, andererseits die Stichprobe dieses Beitrags mit 51 Jugendlichen insgesamt klein ist und auch klinisch unauffällige Jugendliche inkludiert. Weiter enthielt die Stichprobe keine psychotischen Jugendlichen, sodass die IPO-Skala Realitätsprüfung vermutlich kaum zwischen den Jugendlichen differenzierte. Angenommen werden kann weiter, dass auch strukturell nicht beeinträchtigte Jugendlichen altersgemäß eine Verunsicherung ihrer Identität zu meistern haben (z. B. Foelsch et al. 2010; Kernberg et al. 2001). Offensichtlich ist der Kurzfragebogen IPO-16 nicht in der Lage, zwischen einer normalen Identitätskrise und einer gravierenderen Identitätsdiffusion bei Jugendlichen $\mathrm{zu}$ differenzieren. Das Vorherrschen primitiver Abwehr hingegen (mit dem Fokus auf Spaltung) hängt systematisch mit struktureller Beeinträchtigung zusammen und findet sich bei besser strukturierten Jugendlichen deutlich weniger.

Trotz dieser theoretisch sinnvollen korrelativen Zusammenhänge der genannten Fragebogen-Skalen mit den OPD-KJ-Strukturratings, eigneten sich die Fragebögen nur bedingt als Struktur-Screening bei Jugendlichen, wie die Berechnungen der logistischen Regressionen ergaben. Hierbei ist zunächst kritisch zu erwähnen, dass die Stichprobengröße für dieses Verfahren recht klein war. In einer größeren Stichprobe mit einem größeren Anteil klinischer Population hätten vermutlich auch Modelle mit mehr Prädiktoren Bestand gehabt, sodass möglicherweise eine höhere Varianzaufklärung erzielt worden wäre. Im vorliegenden Fall konnten durch reduziertes Erleben positiver Emotionen und hohe Neigung zu Blackout als Reaktion auf subjektiv als schwierig erlebte Gefühle knapp $60 \%$ der strukturell beeinträchtigten Jugendlichen korrekt zugeordnet werden. Dies bedeutet jedoch auch, dass $40 \%$ der strukturell beeinträchtigten Jugendlichen durch dieses Screening nicht erfasst wurden. Würde man rein auf der Basis der Werte dieser Fragebogen-Skalen die Entscheidung für eine ausführliche Strukturdiagnostik mittels OPDInterview treffen, blieben $40 \%$ der hoch-vulnerablen Jugendlichen als solche unerkannt. Weiterführende Forschungsbemühungen in diesem Bereich sind dringend angezeigt, da vermutet werden kann, dass ein solches Screening anhand von Selbstbeurteilungsverfahren, die an einer jugendlichen Population normiert sind, bessere Ergebnisse erzielte.

Dies ist eine wichtige und nicht unwesentliche Limitation dieser Studie. Die hier verwendeten Fragebögen wurden für den Erwachsenenbereich entwickelt, dies führt zu Einschränkungen in der Interpretierbarkeit der Ergebnisse. Aktuell wurde von der Arbeitsgruppe um Schmeck und Goth in Basel (für einen Überblick siehe Schrobildgen et al. 2014) das Assessment of Identity Development and Identity Diffusion in Adolescence entwickelt (AIDA; Goth et al. 2012; Jung et al. 2013). Die ersten Ergebnisse hierzu zeigten, dass sich Borderline-Persönlichkeitsstörungen bei Jugendlichen ab einem bestimmten Ausmaß an Identitätsdiffusion identifizieren ließen. Auch ein Selbstbeurteilungsverfahren (LoPF-QA; Goth 2015) aufbauend auf den Levels of Personality Functioning des DSM-5 ist derzeit in Entwicklung. Zudem wird aktuell an der Universitätsklinik für Kinder- und Jugendpsychiatrie Innsbruck versucht, für den OPD-Strukturfragebogen (OPD-SF; Ehrenthal et al. 2012) eine Adaption an das Jugendalter durchzuführen.

Zusammenfassend zeigen die Ergebnisse der vorliegenden Studie, dass ein Screening durch die gewählten Selbstbeurteilungsverfahren nicht empfohlen werden kann. Auch wenn ein „schnelles RisikoScreening“ für den klinischen Alltag sehr ökonomisch und wünschenswert wäre, lässt sich das klinische Expertenurteil nicht durch den Einsatz von Fragebögen ersetzen.

Danksagung Wir danken allen DiplomandInnen und PraktikantInnen, sowie den unterstützenden KollegInnen, welche an diesem Projekt mitgearbeitet haben. Weiter danken wir den Jugendlichen für ihr Vertrauen und ihre Bereitschaft und Neugier, an dem Forschungsprojekt mitzuwirken.

Open access funding provided by University of Innsbruck and Medical University of Innsbruck.

Förderung Dieses Projekt wurde vom Tiroler Wissenschaftsfond finanziell gefördert durch Drittmittelvergabe an Cord Benecke (Nr.: UNI-0404/559) und Astrid Bock (Nr.: UNI0404/1351).

Interessenkonflikt E. Huber, A. Bock, M. Hans, E. Wieser, M. Steinmayr-Gensluckner, K. Sevecke und C. Benecke geben an, dass kein Interessenkonflikt besteht.

Open Access This article is distributed under the terms of the Creative Commons Attribution 4.0 International License (http://creativecommons.org/licenses/by/4.0/), which permits unrestricted use, distribution, and reproduction in any medium, provided you give appropriate credit to the original author(s) and the source, provide a link to the Creative Commons license, and indicate if changes were made. 


\section{Literatur}

Arbeitskreis-OPD-KJ (2007). Operationalisierten Psychodynamischen Diagnostik im Kindes- und Jugendalter. Grundlagen und Manual (2.Aufl.). Bern:Huber.

Arbeitskreis-OPD-KJ (2013). OPD-KJ-2: Operationalisierte Psychodynamische Diagnostik im Kindes-und Jugendalter: Grundlagen und Manual. Bern:Huber.

Arbeitskreis-OPD (1996). Operationalisierte Psychodynamische Diagnostik - OPD. Grundlagen und Manual. Bern Huber.

Arbeitskreis-OPD (2006). Operationalisierte Psychodynamische Diagnostik - OPD-2. Das Manual für Diagnostik und Therapieplanung. Bern:Huber.

Barlow, D. H., Farchione, T. J., Fairholme, C.P., Ellard, K. K., Boisseau, C. L., Allen, L. B., \& Ehrenreich-May, J. (2011). The unified protocol for transdiangostic treatment of emotional disorders: therapist guide. New York: Oxford University Press.

Barnow, S. (2012). Emotionsregulation und Psychopathologie. Ein Überblick. Psychologische Rundschau, 63, 111-124.

Becker, D., Grilo, C., Morey, L., Walker, M., Edell, W., \& McBlashan, T. (1999). Applicability of personality disorder criteria it hospitalised adolescents: evaluation of internal consistencyand criterion overlap. Journal of the American Academy of Child and Adolescent Psychiatry, 38, 200-205.

Bender, P. K., Reinholdt-Dunne, M. L., Esbjorn, B. H., \& Pons, F. (2012). Emotion dysregulation and anxiety in children and adolescents: gender differences. Personality and Individual Differences, 53, 284-288.

Benecke, C. (2006). Affektivität, Beziehung und psychische Störung - Abschlußbericht für den Jubiläumsfonds der ÖNB. Innsbruck: Institut für Psychologie, Universität Innsbruck.

Benecke, C. (2014). Klinische Psychologie und Psychotherapie. Ein integratives Lehrbuch. Stuttgart: Kohlhammer.

Benecke, C., Bock, A., Wieser, E., Tschiesner, R., Lochmann, M., Küspert, F., Schorn, R., Viertler, B., \& SteinmayerGensluckner, M. (2011). Reliabilität und Validität der OPD-KJ-Achsen Struktur und Konflikt. Praxis der Kinderpsychologie und Kinderpsychiatrie, 60, 60-73.

Benecke, C., Koschier, A., Peham, D., Bock, A., Dahlbender, R. W., Biebl, W., \& Doering, S. (2009). Erste Ergebnisse zu Reliabilität und Validität der OPD-2 Strukturachse. Zeitschrift für Psychosomatische Medizin und Psychotherapie, 1, 84-96.

Benecke, C., Vogt, T., Bock, A., Koschier, A., \& Peham, D. (2008). Emotionserleben und Emotionsregulation und ihr Zusammenhang mit psychischer Symptomatik. Psychotherapie Psychosomatik Medizinische Psychologie, $58,366-370$

Berking, M., Wuppermann, P., Reichardt, A., Pejic, T., Dippel, A., \& Znoj, H. (2008). General emotion-regulation skills as a target in psychotherapy. Behaviour Research and Therapy, 46, 1230-1237.

Bernstein, D. P., Cohen, P., Velez, C. N., Schwab-Stone, M., Siever, L. J., \& Shinsato, L. (1993). Prevalenzand stability of the DSM-III-R personality disorders in a community-based survey of adolescents. American Journal of Psychiatry, $150,1237-1243$

Bifulco, A., Moran, P. M., Ball, C., Jacobs, C., Baines, R., Bunn, A., \& Cavagin, J. (2002). Childhood adversity, parental vulnerability and disorder: examining inter-generational transmission of risk. Journal of Child Psychology and Psychiatry, 43, 1075-1086.

Bouchard, M.-A., Target, M., Lecours, S., Fonagy, P., Tremblay, L.-M., Schachter, A., \& Stein, H. (2008). Mentalization in adult attachment narratives: Reflective functioning, mental states, and affect elaboration compared. Psychoanalytic Psychology, 25, 47-66.

Carter, J.D., Joyce, P. R., Mulder, R. T., \& Luty, S. E. (2001). The contribution of temperament, childhood neglect, and abuse to the development of personality dysfunction: a comparison of three models. Journal of Personality Disorders, 15, 123-135.

Cimbora, C. M., \&McIntosh, D. M. (2003).Emotional responses to antisocial acts in adolescent males with conduct disorder: a link to effective morality. Journal of Clinical Child and Adolescent Psychology, 32, 296-301.

Crawford, T. N., Cohen, P., First, M. B., Skodol, A. E., Johnson, J.G., \& Kasen, S. (2008). Comorbid axis I and axis II disorders in early adolescence. outcomes 20 years later. Archives of General Psychiatry, 65, 641-648.

Cropp, C., Salzer, S., Häusser, L. F., \& Streeck-Fischer, A. (2013). Interrater-Reliabilität und Konstruktvalidität der OPDKJ-Achse Struktur - Erste Forschungsergebnisse zum Einsatz von OPD-KJ im Rahmen klinischer Routine. Praxis der Kinderpsychologie und Kinderpsychiatrie, 62, 270-284.

Crowell, S.E., Baucom, B. R., Yaptangco, M., Bride, D., Hsiao, R., McCauley, E., \& Beauchaine, T.P. (2014). Emotion dysregulation and dyadic conflict in depressed and typical adolescents: evaluating concordance across psychophysiological and observational measures. Biological Psychology, 98, 50-58.

Dubo, E.D., Zanarini, M.C., Lewis, R.E., \& Williams, A.A. (1997). Childhood antecedents of self-destructiveness in borderline personality disorder. Canadian Journal of Psychiatry, 42, 63-69.

Egle, U.T., Hoffmann, S. O., \& Joraschky, P. (Hrsg.). (2005). Sexueller Missbrauch, Misshandlung, Vernachlässigung. Erkennung, Therapie und Prävention der Folgen früher Stresserfahrungen (3. Aufl.). Stuttgart: Schattauer.

Ehrenthal, J.C., Dinger, U., Horsch, L., Komo-Lang, M., Klinkerfuß, M., Grande, T., \& Schauenburg, H. (2012). Der OPD-Strukturfragebogen (OPD-SF): Erste Ergebnisse zu Reliabilität und Validität. Psychotherapie, Psychosomatik, Medizinische Psychologie, 62, 25-32.

Farrington, D. P. (2000). Psychosocial predictors of adult antisocial personality and adult convictions. Behavioral Sciences and the Law, 18, 605-622.

Foelsch, P.A., Odom, A., Arena, H., Krischer, M. K., Schmeck, K., \& Schlüter-Müller, S. (2010). Differenzierung zwischen Identitätskrise und Identitätsdiffusion und ihre Bedeutung für die Behandlung. Praxis der Kinderpsychologie und Kinderpsychiatrie, 59, 418-434.

Fonagy, P., Gergely, G., Jurist, E. L., \& Target, M. (2002). Affect regulation, mentalization and the development of the self. New York: Other Press.

Fonagy, P., Leigh, T., Steele, M., Steele, H., Kennedy, R., Mattoon, G., Target, M., \& Gerber, A. (1996). The relation of attachment status, psychiatric classification, and response to psychotherapy. Journal of Consulting and Clinical Psychology, 64, 22-31.

Fossati, A., Feeney, J., Maffei, C., \& Borroni, S. (2014). Thinking about feelings: affective sate mentalization, attachment styles, and borderline personality disorder features among italian nonclinical adolescents. Psychoanalytic Psychology, 31, 41-67.

Fossati, A., Gratz, K. L., Maffei, C., \& Borroni, S. (2013). Emotion dysregulation and impulsivity additively predict borderline personality disorder features in Italian nonclinical adolescents. Personality and Mental Health, 7, 320-333. 
Fussner, L. M., Luebbe, A. M., \& Bell, D. J. (2015). Dynamic of positive emotion regulation: associations with youth depressive symptoms. Journal of Abnormal Child Psychology, 43, 475-488.

Fydrich, T., Renneberg, B., Schmitz, B., \& Wittchen, H.-U. (1997). SKID-II. Strukturiertes Klinisches Interview für DSM-IV. Achse II: Persönlichkeitsstörungen. Göttingen: Hogrefe.

Gilbert, K.E. (2012). The neglected role of positive emotion in adolescent psychopathology. Clinical Psychology Review, $32,467-481$

Gladstone, G., Parker, G., Wilhelm, K., Mitchell, P., \& Austin, M.P. (1999). Characteristics of depressed patients who report childhood sexual abuse. American Journal of Psychiatry, 156, 431-437.

Golier, J. A., Yehuda, R., Bierer, L. M., Mitropoulou, V., New, A. S., Schmeidler, J., Silverman, J. M., \& Siever, L. J. (2003). The relationship of borderline personality disorder to posttraumatic stress disorder and traumatic events. American Journal of Psychiatry, 160, 2018-2024.

Goth, K. (2015). Beschreibung der „Persönlichkeitsfunktionen und Struktur" - Haupttestfragebögen (LoPF-QA). unveröffentlichtes Manuskript

Goth, K., Foelsch, P.A., Schlüter-Müller, S., \& Schmeck, K. (2012). AIDA: a self report questionnaire for measuring identity in adolescents - short manual. Basel: Child and Adolescent Psychiatric Hospital, Psychiatric University Hospitals.

Greuel, J. F, Reinhold, N., Wenglorz, M., \& Heinrichs, N. (2015). Selbstberichtete Strategien zur Emotionsregulation bei Kindern und Jugendlichen mit psychischen Störungen. Praxis der Kinderpsychologie und Kinderpsychiatrie, 64, 368-385.

Grilo, C. M., \& Masheb, R. M. (2002). Childhood maltreatment and personality disorders in adult patients with binge eating disorder. Acta Psychiatrica Scandinavica, 106, 183-188.

Ha, C., Sharp, C., Ensink, K., Fonagy, P., \& Cirino, P. (2013). The measurement of reflective function in adolescents with and without borderline traits. Journal of Adolescence, 36, 1215-1223.

Helmsen, J., Koglin, U., \& Petermann, F. (2012). Emotion regulation and aggressive behavior in preeschoolers: the mediating role of social information processing. Child Psychiatry And Human Development, 43, 87-101.

Herpertz-Dahlmann, B., Resch, F., Schulte-Markwort, M., \& Warnke, A. (2003). Entwicklungspsychiatrie. Stuttgart: Schattauer.

Highfield, J., Markham, D., Skinner, M., \& Neal, A. (2010). An investigation into the experience of self-conscious emotions in individuals with bipolar disorder, unipolar depression and non-psychiatric controls. Clinical Psychology and Psychotherapy, 17,395-405.

In-Albon, T., Tschan, T., Schwarz, D., \& Schmid, M. (2015). Emotionsregulation bei Jugendlichen mit Nichtsuizidalen Selbstverletzungen. Praxis der Kinderpsychologie und Kinderpsychiatrie, 64,386-403.

Jelen-Mauboussin, A., Klipsch, O., Pressel, C., Lenz, K., Lehmkuhl, U., \& Winter, S. (2013). Operationalisierte Psychondynamische Diagnostik des Kindes- und Jugendalters. Veränderungsmessungen mit der Strukturachse bei Kindern und Jugendlichen mit psychiatrischer Störung. Psychotherapeut, 58,24-30.

Johnson, J. G., Cohen, P., Kasen, S., Skodol, A. E., Hamagami, F., \& Brook, J. S. (2000). Age-related change in personality disorder trait levels between early adolescence and adult- hood: a community-based longitudinal investigation. Acta Psychiatrica Scandinavica, 102, 265-275.

Johnson, J. G., Cohen, P., Kasen, S., Skodol, A. E., \& Oldham, J. M. (2008). Comulative prevalence of personality disorders between adolescence and adulthood. Acta Psychiatrica Scandinavica, 118, 410-413.

Joyce, P. R., McKenzie, J. M., Luty, S. E., Mulder, R. T., Carter, J. D., Sullivan, P.F., \& Cloninger, C. R. (2003). Temperament, childhood environment and psychopathology as risk factors for avoidant and borderline personality disorders. Australian an New Zealand Journal of Psychiatry, 37, 756-764.

Jung, E., Pick, O., Schlüter-Müller, S., Schmeck, K., \& Goth, K. (2013). Identity development in adolescents with mental problems. Child and Adolescent Psychiatry and Mental Health, 7, 1-8.

Kämmerer, A. (2010). Zur Intensität des Erlebens von Schamgefühlen bei psychischen Störungen. Psychotherapie, Psychosomatik, Medizinische Psychologie, 60, 262-270.

Kasen, S., Cohen, P., Skodol, A.E., Johnson, J.G., \& Brook, J.S. (1999). Influence of child and adolescent psychiatric dosorders an young adult personality disorder. American Journal of Psychiatry, 156, 1529-1535.

Kashdan, T.B., Farmer, A.S., Adams, L. M., Ferssizidis, P., McKnight, P.E., \& Nezlek, J.B. (2013). Distinguishing healthy adults from people with social anxiety disorder: evidence for the value of experiential avoidance and positive emotions in everyday social interactions. Journal of Abnormal Psychology, 122, 645-655.

Kernberg, O.F. (1992). Schwere Persönlichkeitsstörungen (4.Aufl.). Stuttgart: Klett-Cotta.

Kernberg, O. F., \& Caligor, E. (2005). A psychoanalytic theory of personality disorders. In M. F. Lenzenweger, \& J. F. Clarkin (Hrsg.), Major theories of personality disorder (2. Aufl. S. 114-156). NewYork: Guilford Press.

Kernberg, P.F., Weiner, A.S., \& Bardenstein, K.K. (2001). Persönlichkeitsstörungen bei Kindern und Jugendlichen. Stuttgart: Klett-Cotta.

Kiene, V. (2007). Psychische Bedingungen von Aggression und Gewalt: eine empirische Untersuchung zum Vergleich aggressiver und nicht-aggressiver Kinder bei Fremdunterbringung. Unveröffentl. Dissertation, Universität Innsbruck.

Kim, S., Sharp, C., \& Carbone, C. (2014). The protective role of attachment security for adolescent borderline personality disorder features via enhanced positive emotion regulation strategies. Personality Disorders: Theory, Research, and Treatment, 5, 125-136.

Krischer, M. K., Sevecke, K., Döpfner, M., \& Lehmkuhl, G. (2006). Persönlichkeitsstörungsmerkmale im Kindesund Jugendalter: Konzepte, methodische Ansätze und empirische Ergebnisse. Zeitschrift für Kinder- und Jugendpsychiatrie und Psychotherapie, 34, 87-100.

Kullik, A., \& Petermann, F. (2013). Dysfunktionale Emototionsregulation als grundlegendes Merkmal von Jugendlichen mit Angst- und depressiver Störung. Fortschritte der Neurologie - Psychiatrie, 81,35-39.

Lange, S., \& Tröster, H. (2014). Strategien der Emotionsregulation bei sozial ängstlichen Kindern und Jugendlichen. Zeitschrift für Klinische Psychologie und Psychotherapie, 43,35-42.

Laucht, M., Esser, G., \& Schmidt, M.H. (1992). Psychisch auffällige Eltern: Risiken für die kindliche Entwicklung im Säuglings und Kleinkindalter? Zeitschrift für Familienfor schung, 4,22-48.

Lehmkuhl, G. (2006). Entwicklung von Persönlichkeitsmerkmalen und Persönlichkeitsstörungen im Kindes- und 
Jugendalter. In A. Remmel, O. F. Kernberg, W. Vollmoeller, \& B. Strauß (Hrsg.), Handbuch Körper und Persönlichkeit. Entwicklungspsychologie, Neurobiologie und Therapie von Persönlichkeitsstörungen (S. 91-101). Stuttgart: Schattauer.

Leising, D., Rudolf, G., \& Grande, T. (2004). Die Klinische Emotionsliste - ein Verfahren zur Erfassung des affektiven Erlebensrepertoires von Patienten. Psychotherapie, Psychosomatik, Medizinische Psychologie, 54, 173-178.

Lenzenweger, M. F. (1997). Stability and change in personality disorder features: the longitudinal study of personality disorders. Archives of General Psychiatry, 56, 1009-1015.

Léonard, S., Steiger, H., \& Kao, A. (2003). Childhood and adulthood abuse in bulimic and nonbulimic women: prevalences and psychological correlates. International Journal of Eating Disorders, 33, 397-405

Löwe, B., Spitzer, R. L., Zipfel, S., \& Herzog, W. (2002). Gesundheitsfragebogen für Patienten (PHQ D). Komplettversion und Kurzform. Testmappe mit Manual, Fragebögen, Schablonen (2. Aufl.). Karlsruhe: Pfizer.

Marsh, A.A., Finger, E. C., Schechter, J.C., Jurkowitz, I.T. N., Reid, M. E., \&Blair, R. J. R. (2011).Adolescents with psychopathic traits report reduction in physiological resonses to fear. Journal of Child Psychology and Psychiatry, 52, 834-841.

Mathews, B. L., Kerns, K. A., \& Ciesla, J. A. (2014). Specificity of emotion regulation difficulties related to anxiety in early adolescence. Journal of Adolescence, 37, 1089-1097.

Mattanah, J.J.F., Becker, D.F., Levy, K. N., Edell, W.S., \& McGlashan, T.H. (1995). Diagnostic stability in adolescents followed up 2 years after hospitalisation. American Journal of Psychiatry, 152, 889-894.

Meijer, M., Goedhart, A. W., \&Treffers, P. D. A. (1998).The persistence of borderline personality disorder in adolescence. Journal of Personality Disorders, 12, 13-22.

Mills, P., Newman, E. F., Cossar, J., \& Murray, G. (2015). Emotional maltreatment and disordered eating in adolescents: testing the mediating role of emotion regulation. Child Abuse and Neglect, 39, 156-166.

Mulder, R. T., Joyce, P. R., \& Cloninger, C. R. (1994). Temperament and early environment influence comorbidity and personality disorders in major depression. Comprehensive Psychiatry, 35, 225-233.

Myin-Germeys, I., Peeters, F., Havermans, R., Nicolson, N. A., DeVries, M. W., Delespaul, P., \&Van Os, J. (2003).Emotional reactivity to daily life stress in psychosis and affective disorder: an experience sampling study. Acta Psychiatrica Scandinavica, 107, 124-131.

Petermann, F., \& Niebank, K. (1999). Entwicklungspsychopathologie- Konzepte und Ergebnisse. Psychotherapeut, 44, 257-264.

Queens, A. H., \& Ehrenreich-May, J. (2014). Anxiety-disordered adolescents with and without comorbid depressive disorder: variations in clinical presentation and emotion regulation. Journal of Emotional and Behavioral Disorders, 22, 160-170.

Rudolf, G. (2002). Strukturbezogene Psychotherapie. In G. Rudolf, T. Grande, \& P. Henningsen (Hrsg.), Die Struktur der Persönlichkeit(S. 249-271). Heidelberg: Schattauer.

Rudolf, G., \& Henningsen, P. (Hrsg.). (2008). Psychotherapeutische Medizin und Psychosomatik. Ein einführendes Lehrbuch aud psychodynamischern Grundlage (6. Aufl.). Stuttgart:Thieme.

Schenkel, L. S., Marlow-O'Connor, M., Moss, M., Sweeney, J.A., \& Pavuluri, M.N. (2008). Theory of mind and social inference in children and adolescents with bipolar disorder. Psychological Medicine, 38, 791-800.
Schramm, A. T., Venta, A., \& Sharp, C. (2013). The role of experiential avoidance in the association between borderline features and emotion regulation in adolescents. Personality Disorders: Theory, Research, and Treatment, 4, 138-144.

Schrobildgen, C., Birkhälzer, M., Schmeck, K., \&Goth, K. (2014). Diagnostik von Identitäts- und Persönlichkeitsstörungen im Jugendalter. Psychiatrie \& Neurologie, 2, 9-12.

Schulte-Markwort, M., Diepold, B., \& Resch, F. (Hrsg.). (2001). Psychische Störungen bei Kindern und Jugendlichen (2.Aufl.). Stuttgart: Thieme.

Scott, L.N., Stepp, S.D., Hallquist, M.N., Whalen, D.J., Wright, A. G.C., \& Pilkonis, P.A. (2015). Daily shame and hostile irritability in adolescent girls with borderline personality disorder symptoms. Personality Disorders: Theory, Research, and Treatment, 6, 53-63.

Seiffge-Krenke, I., Mayer, S., Rathgeber, M., \& Sommer, T. (2013). Konflikt- und Strukturachse der Operationalisierten Psychodynamischen Diagnostik des Kindes- und Jugendalters. Hilfe bei Indikation und Therapieplanung. Psychotherapeut, 58, 6-14.

Sevecke, K., \& Krischer, M. K. (2011). Aktuelle Entwicklungslinien in der Persönlichkeitsforschung im Jugendalter. Forensische Psychiatrie, Psychologie, Kriminologie, 5, 154-164.

Sevecke, K., Schmeck, K., \& Krischer, M.K. (2014). Das dimensional-kategoriale Hybridmodell für Persönlichkeitsstörungen im DSM-5 aus jugendpsychiatrischer Perspektive. Zeitschrift für Kinder- und Jugendpsychiatrie und Psychotherapie, 42, 279-283.

Shakoor, S., Jaffee, S. R., Bowes, L., Ouellet- Morin, I., Andreou, P., Happé, F., Moffitt, T.E., \& Arseneault, L. (2012). A prospective longitudinal study of children's theory of mind and adolescent involvement in bullying. Journal of Child Psychology and Psychiatry, 53, 254-261.

Sharp, C., Pane, H., Ha, C., Venta, A., Patel, A.B., Sturek, J., \& Fonagy, P. (2011). Theory of mind and emotion regulation difficutlies in adolescents with borderline traits. Journal of the American Academy of Child and Adolescent Psychiatry, 50, 563-573.

Stefini, A., Reich, G., Horn, H., Winkelmann, K., Ohmes, U., Frost, U., \& Kronmüller, K.-T. (2013). InterraterReliabilität der OPD-KJ Achsen Konflikt und Struktur. Praxis der Kinderpsychologie und Kinderpsychiatrie, 62, 255-269.

Steinberg, L. (2005). Cognitive and affective development in adolescence. Trends in Cognitive Science, 9, 69-74.

Taubner, S., \& Sevecke, K. (2015). Kernmodell der Mentalisierungsbasierten Therapie. Psychotherapeut, 60, 169-184.

Taubner, S., White, L. O., Zimmermann, J., Fonagy, P., \& Nolte, T. (2013). Attachment-related Mentalization moderates the relationship between psychpathic traits and proactive aggression in adolescents. Journal of Abnormal Child Psychology, 41, 929-938.

Taubner, S., Wiswede, D., Nolte, T., \& Roth, G. (2010). Mentalisierung und externalisierende Verhaltensstörungen in der Adoleszenz. Psychotherapeut, 55, 312-320.

Van Durme, K., Braet, C., \& Goossens, L. (2015). Insecure attachmentand eatingpathologyin earlyadolescence: the role of emotion regulation. Journal of Early Adolescence, $35,54-78$.

Venta, A., Hart, J., \& Sharp, C. (2012). The relation between experientail avoidance, alexithymia and emotion regulation in inpatient adolescents. Clinical Child Psychology, 18, 398-410.

Voon, D., Hasking, P., \& Martin, G. (2014a). Change in emotion regulation strategy use and its impact on adolescent 
nonsuicidal self-injury: a three-year longitudinal analysis using latent growth modeling. Journal of Abnormal Psychology, 123, 487-498.

Voon, D., Hasking, P., \& Martin, G. (2014b). Emotion regulation in first episode adolescen non-suicidal self-injury: What difference does a year make? Journal of Adolescence, 37, 1077-1087.

Weitkamp, K., Wiegand-Grefe, S., \& Romer, G. (2013). Reliabilität und Konstruktvalidität der OPD-KJ Achsen Struktur und Behandlungsvoraussetzungen. Praxis der Kinderpsychologie und Kinderpsychiatrie, 62, 243-254.

Yuan, J. W., \& Kring, A. M. (2009). Dysphoria and the prediction and experience of emotion. Cognition and Emotion, 23, 1221-1232.

Zanarini, M. C., Williams, A. A., Lewis, R. E., Reich, R. B., Vera, S. C., Marino, M. F., Levin, A., Yong, L., \& Frankenburg, F. R. (1997). Reported pathological childhood experience associated with the development of borderline personality disorder. American Journal of Psychiatry, 154, 1101-1106.

Zhao, Y., \&Zhao, G. (2015). Emotion regulation and depressive symptoms: examining the mediation effects of school connectedness in chinese late adolescents. Journal of Adolescence, 40, 14-23.
Zimmermann, J., Benecke, C., Hörz-Sagstätter, S., \& Dammann, G. (2015). Normierung der deutschsprachigen 16Item-Version des Inventars der Persönlichkeitsorganisation (IPO-16). Zeitschrift für Psychosomatische Medizin und Psychotherapie, 61, 5-18.

Zimmermann, J., Benecke, C., Hörz, S., Rentrop, M., Peham, D., Bock, A., Wallner, T., Schauenburg, H., Frommer, J., Huber, D., Clarkin, J. F., \& Dammann, G. (2013). Validierung einer deutschsprachigen 16-Item-Version des Inventars der Persönlichkeitsorganisation (IPO-16). Diagnostica, 59, 3-16.

Zimmermann, J., Ehrenthal, J. C., Cierpka, M., Schauenburg, H., Doering, S., \& Benecke, C. (2012). Assessing the level of structural integration using Operationalized Psychodynamic Diagnosis (OPD): implications for DSM5. Journal of Personality Assessment, 94, 522-532.

Zimmermann, J., Ehrenthal, J.C., Hoerz, S., Rentrop, M., Rost, R., Schauenburg, H., Schneider, W., Waage, M., \& Cierpka, M. (2010). Neue Validierungsstudien zur Operationalisierten Psychodynamischen Diagnostik (OPD-2). Psychotherapeut, 55, 69-73. 